\title{
The interacting Fock space of Haldane's exclusion statistics
}

\author{
L. Accardi and M. Nhani
}

October 1, 2006

\begin{abstract}
We prove that Haldane exclusion statistics (ES) can be included in the more general class of standard interacting Fock spaces (IFS). This result provides a standard rule to assign in a consistent way the parameters of the most general exclusion statistics and, through the results of [4], it shows that there is a canonical one-to-one correspondence between these parameters and the families of orthogonal polynomials associated to an arbitrary measure with moments of all orders. Such a correspondence has been discovered in a multiplicity of particular cases (see for example [5], [9]). Thus our result gives a rule to identify immediately the family of orthogonal polynomials associated to a given ES. Moreover it allows to extend the ES in a natural way to infinitely many species and in particular it suggests a standard way to take the thermodynamic limit of these statistics.
\end{abstract}

\section{Introduction}

Let us begin by a brief exposition of the main idea on Haldane exclusion statistics (ES) [6] as described in [2]. In Quantum Statistical Mechanics (QSM) any statistics is described by a partition function. Consider a system of $N$ identical particles, each of which can occupy $K$ independent states corresponding to energies $\varepsilon_{1} \ldots \varepsilon_{K}$ (alternatively we may think of the index $j=1, \ldots, K$ as labeling sites or species, ...). For such a system the partition function has the following structure:

$$
Q_{N}\left(x_{1} \ldots x_{K}\right)=\sum_{\substack{n_{i} \in \mathbb{N} \\ n_{1}+\ldots+n_{K}=N}} \alpha_{N}\left(n_{1} \ldots n_{K}\right) x_{1}^{n_{1}} \ldots x_{K}^{n_{K}}(I)
$$

where $x_{i} \equiv \exp \left(-\varepsilon_{i} / \varepsilon_{T}\right), i=1 \ldots K$. A $K$-uple $\left(n_{1}, \ldots, n_{K}\right)$ of natural integers, i.e.a sequence of occupation numbers for the different states, will be called a configuration. The function $\alpha\left(n_{1} \ldots n_{K}\right)$ which weights all possibles configuration has the following properties (see [1]):

(a) $\alpha\left(n_{1} \ldots n_{K}\right) \geq 0$ 
(b) $\alpha\left(n_{1} \ldots n_{K}\right)$ is a symmetric function [ensuring that no particular $\varepsilon_{i}$ is preferred].

A function $\alpha: \mathbb{N}^{K} \rightarrow \mathbb{R}$ satisfying these properties defines the statistics which the identical particles obey. In the case of Haldane's ES the function $\alpha\left(n_{1} \ldots n_{K}\right)$ must satisfy two additional conditions:

(c) $\alpha\left(n_{1} \ldots n_{i}+1 \ldots n_{K}\right) \leq \alpha\left(n_{1} \ldots n_{i} \ldots n_{K}\right) \forall i=1 \ldots K$

(d) there exists a number $p \in \mathbb{N}$ such that $\alpha\left(p+1,, n_{2} \ldots n_{K}\right)=0$ and $\alpha\left(n_{1} \ldots n_{K}\right)>0$ if $\max \left\{n_{i}: i \leq K\right\} \leq p$.

Ilinski, Gunn and Ilinskaia (see [2]) have constructed an Hilbert space $\mathcal{H}$, called fractional-dimensional Fock space (FDFS), with the property that (1) is a weighted partition function

$$
Z=Q_{N}\left(x_{1} \ldots x_{K}\right)=\operatorname{Tr}\left(f(N) e^{-\beta H}\right)
$$

where the trace is meant in $\mathcal{H}$,

$$
H=\sum_{j} \varepsilon_{j} N_{j}
$$

is the usual Hamiltonian of an ideal gas, $N_{j}(j=1, \ldots, K)$ is the number operator counting the number of particles in the $j$-th species) and $f(N)$ (denoted $I d$ in [2]) is a self-adjoint operator (a function of the occupation numbers of each species) characterizing the ES. The trace of the operator $I d$ (always finite because of property $(d)$ above) has been called in [2] the fractional dimension of the Hilbert space of these configurations.

We show in this paper that the fractional-dimensional Fock space is in fact a particular case of a standard interacting Fock space.

\section{One mode interacting Fock spaces (IFS)}

In this section we recall some properties of the one mode interacting Fock spaces and we refer to the survey paper [3] for a discussion of the general case. The one mode interacting Fock spaces $\Gamma(\mathbb{C})$ is defined as follows: as a vector space $\Gamma(\mathbb{C})$ is the direct sum of 1 -dimensional sub-spaces

$$
\Gamma(\mathbb{C})=\bigoplus_{n=0}^{\infty} \mathbb{C} \mid n>
$$

The space $\mathbb{C} \mid n>$ is called the $n$-particle sub-space. On $\Gamma(\mathbb{C})$ one defines a Hilbert space structure by prescribing that: (i) the sum in (1) is orthogonal, i.e. different $n$-particle sub-spaces are orthogonal; (ii) the norm of the vector $|n\rangle$ is given by

$$
\langle n \mid n\rangle=\lambda_{n}
$$


(iii) the creation operator is well defined by the prescription

$$
a^{+}|n>=| n+1>
$$

Notice that, because of (2), condition (iii) implies that, if $\lambda_{n}$ is zero, then all $\lambda_{k}$ with $k>n$ must also be zero Therefore the adjoint of the creation operator (annihilator) is uniquely defined by the condition:

$$
a\left|n+1>=\frac{\lambda_{n+1}}{\lambda_{n}}\right| n>
$$

with the convenction that $0 / 0=0$. Moreover one has

$$
\begin{gathered}
\langle n, n\rangle=\left\langle a^{+}(n-1), n\right\rangle=\langle(n-1), a n\rangle=\frac{\lambda_{n}}{\lambda_{n-1}}\langle n-1, n-1\rangle=\ldots \\
\|n>\|^{2}=\frac{\lambda_{n}}{\lambda_{n-1}} \cdot \frac{\lambda_{n-1}}{\lambda_{n-2}} \ldots \frac{\lambda_{1}}{\lambda_{0}}=\frac{\lambda_{n}}{\lambda_{0}}
\end{gathered}
$$

which, together with (2) gives $\lambda_{0}=1$.

\section{Inclusion of the one mode FDFS into the one mode IFS}

In order to establish the connection between IFS, orthogonal polynomyals (see [4]) and the ES it is useful to introduce the numbers

$$
\omega_{j}=\frac{\lambda_{j}}{\lambda_{j-1}} ; \quad \lambda_{0}=1
$$

so that

$$
\lambda_{n}=\prod_{j=0}^{n} \omega_{j}
$$

It is clear that the sequence $\left(\omega_{j}\right)$ uniquely determines the IFS. Moreover

$$
\begin{gathered}
a\left|n+1>=\omega_{n+1}\right| n> \\
a^{+} a\left|n>=\omega_{n}\right| n>
\end{gathered}
$$

In [2] the additional condition

$$
N\left|n>=a_{0}^{+} a_{0}\right| n>=n \mid n>
$$

is required. In order to satisfy also this condition we introduce a new creation operator $a_{0}^{+}$, defined by the condition:

$$
a_{0}^{+}\left|n>:=\alpha_{n}\right| n+1>=\alpha_{n} a^{+} \mid n>
$$


thus, in the notation (1):

$$
a_{0}^{+}=a^{+} \alpha_{N}
$$

since $N$, hence $\alpha_{N}$, is self-adjoint also for the interacting scalar product, the adjoint of $a_{j}^{+}$is

$$
a_{0}=\alpha_{N} a
$$

Therefore

$$
\begin{gathered}
a_{0}^{+} a_{0}\left|n>=a_{0}^{+} \alpha_{N} a\right| n>=\omega_{n} a_{0}^{+} \alpha_{N}\left|n-1>=\omega_{n} \alpha_{n-1} a^{+} \alpha_{N}\right| n-1>= \\
\omega_{n} \alpha_{n-1}^{2} a^{+}\left|n-1>=\omega_{n} \alpha_{n-1}^{2}\right| n>
\end{gathered}
$$

If we want

$$
a_{0}^{+} a_{0}=N
$$

we must then have

$$
\omega_{n} \alpha_{n-1}^{2}=n
$$

or

$$
\alpha_{n-1}=\left(\frac{n}{\omega_{n}}\right)^{1 / 2}
$$

Equivalently

$$
\alpha_{n}=\left(\frac{n+1}{\omega_{n+1}}\right)^{1 / 2}
$$

which, given (2) below, coincides with the result of [2]. Summing up:

LEMma (1) The FDFS of [2] (hence Haldane ES in the case of a single species) is isomorphic to the IFS uniquely defined by the prescriptions:

$$
\omega_{n+1}=1-g n
$$

and $\omega_{n+1}=0$ as soon as the right hand side of (2) becomes negative.

ProOF From the above discussion.

REMARK The right hand side of (2) shows why in the Haldane statistics we must restrict to finite dimensional spaces. In fact if $n>1 / g$ the right hand side of (2) becomes negative and this is incompatible with the positivity of the scalar product which requires

$$
\omega_{n} \geq 0 ; \quad \forall n
$$

The general connection between the $\omega_{n}$ 's and the Jacobi coefficients of orthogonal polynomials is described in [4]. 


\section{IFS for many species}

We fix a number $K<+\infty$, representing the number of species, (the case $K \leq$ $+\infty$ can be treated in a similar way) and, for each $j=1, \ldots, K$ let be given a one mode IFS $\left\{\Gamma(\mathbb{C}),\left(\lambda_{n}^{(j)}\right)_{n}\right\}$. On the algebraic tensor product of vector spaces

$$
\mathcal{H}=\otimes{ }^{K} \Gamma(\mathbb{C})=\Gamma(\mathbb{C}) \otimes \ldots \otimes \Gamma(\mathbb{C})
$$

we consider the Hilbert space structure induced by the tensor product of the scalar products defined by the $\left(\lambda_{n}^{(j)}\right)_{n}$ as explained in section (??). Then we define the global $N$-particle structure as follows: we refer each IFS

$$
\left\{\Gamma(\mathbb{C}),\left(\lambda_{n}^{(j)}\right)_{n}\right\}
$$

to an orthonormal basis

$$
\mid n_{j}>\quad ; \quad n_{j} \in \mathbb{N} ; \quad j=1, \ldots, K
$$

and we define the global $N$-particle space by

$$
\mathcal{H}_{N}=\bigoplus_{n_{1}+\ldots+n_{K}=N} \mathbb{C}\left|n_{1}>\otimes \ldots \otimes\right| n_{K}>
$$

an orthonormal basis of $\mathcal{H}_{N}$ is

$$
\left|n_{1}, \ldots, n_{K}>=\right| n_{1}>\otimes \ldots \otimes \mid n_{K}>; \quad n_{1}+\ldots+n_{k}=N
$$

Introducing the multiple number operator

$$
N:=N_{1} \otimes \ldots \otimes N_{K}
$$

where $N_{j}(j=1, \ldots, K)$ is the number operator of the $j$-th species in $\mathcal{H}$ :

$$
N_{j}\left|n_{1}, \ldots, n_{K}>=n_{j}\right| n_{1}, \ldots, n_{K}>
$$

and the operator

$$
W_{N}:=\sum_{n_{1}+\ldots+n_{K}}\left[\prod_{i=1}^{K} \lambda_{n_{i}}^{(i)}\right]\left|n_{1}, \ldots, n_{K}\right\rangle\left\langle n_{1}, \ldots, n_{K}\right|
$$

we see that the scalar product on $\mathcal{H}_{N}$ is defined by

$$
\langle\xi, \eta\rangle_{\mathcal{H}_{N}}=\left\langle\xi, W_{N} \eta\right\rangle_{\otimes^{N} \Gamma(\mathbb{C})}=\left\langle\xi, W_{N} \eta\right\rangle_{\otimes^{N} \Gamma(\mathbb{C})}
$$

where $\langle\xi, \cdot \eta\rangle_{\otimes^{N} \Gamma(\mathbb{C})}$ denotes the usual scalar product on $\otimes^{N} \Gamma(\mathbb{C})$ (i.e. the one coming from the tensor product of the usual scalar products). With these notations it is easy to see that

$$
\mathcal{H}:=\bigoplus_{N \geq 0} \mathcal{H}_{N}
$$


is a standard IFS with weights $W_{N}$ given by (1). The fact that the operator $W_{N}$ does not factorize: this fact corresponds to an interaction, among the different species, of a purely statistical nature, i.e. not encoded into the dynamics, and justifies the name interacting Fock space.

REMARK A more general interacting Fock space can be obtained by replacing, in (1), the operator $W_{N}$ by

$$
W_{N}:=\sum_{n_{1}+\ldots+n_{K}=N} \alpha_{N}\left(n_{1}, \ldots, n_{K}\right)\left|n_{1}, \ldots, n_{K}><n_{K}, \ldots, n_{1}\right|
$$

where $\alpha$ is a function of $\left(n_{1}, \ldots, n_{K}\right)$. In the notations of [2], the operator $I d$ is the direct sum of all the operators $W_{N}$. The case (1) corresponds to the factorization

$$
\alpha_{N}\left(n_{1}, \ldots, n_{K}\right)=\prod_{i=1}^{K} \alpha\left(n_{i}\right)
$$

\section{The IFS of Haldane's ES: the case of many one mode species}

Consider now a system of identical particles obeying Haldane's ES with parameter $g$. We suppose first that $g=\frac{1}{p}, p \geq 1$. Let $K \in N$ be the dimension of the states Hilbert space $H$ of a single-particle. As we said in the introduction we can as well say that we have $K$ species of particles $j=1, \ldots, K$ each of which has a state space of dimension $1: H_{j}=C e_{j}$. All the species having the same statistical parameter $g$. To each species $j=1, \ldots, K$ we associate the IFS $\left\{\Gamma(\mathbb{C}),\left(\lambda_{n}^{(j)}\right)_{n}\right\}$ as in section $(4)$. We define the Hilbert space of the system with $K$ species to be the space introduced in section (4), i.e.:

$$
\mathcal{H}:=\otimes \Gamma(C)=\Gamma_{1} \otimes \Gamma_{2} \otimes \ldots \otimes \Gamma_{K}
$$

Introducing the global $N$-particle spaces as in section (4), we define the scalar products:

$$
\forall N \in \mathbb{N}, \quad \forall u, v \in H_{N} \quad(u, v)_{N}=\left\langle W_{N}(u), v\right\rangle_{N}
$$

where $\langle\cdot\rangle_{N}$ is the natural scalar product on $H_{N}$ and

$$
W_{N}\left|n_{1}, \ldots, n_{K}\right\rangle=\lambda_{n_{1}}^{(1)} \lambda_{n_{2}}^{(2)} \ldots \lambda_{n_{k}}^{(k)}\left|n_{1}, \ldots, n_{K}\right\rangle
$$

\section{REMARKS:}

(i) The function $\alpha: \mathbb{N}^{K} \rightarrow \mathbb{R}$ which weights the different occupation numbers in the partition function (1.1) is determined by the sequences $\left(\lambda_{n_{j}}^{(j)}, n_{j}=\right.$ $1 \ldots p), j=1, \ldots, K$, by:

$$
\alpha\left(n_{1} \ldots n_{K}\right)=\lambda_{n_{1}}^{(1)} \lambda_{n_{2}}^{(2)} \ldots \lambda_{n_{K}}^{(K)}, \quad \forall\left(n_{1} \ldots n_{K}\right) \in \mathbb{N}^{K}
$$


(ii) In the Ilinski, Gunn, Ilinskaia's interpretation of Haldane's ES the $\lambda_{n_{j}}^{(j)}$ are given by:

$$
\lambda_{n_{j}}^{(j)}=(1-g)(1-2 g) \ldots\left(1-\left(n_{j}-1\right) g\right), \quad j=1 \ldots K
$$

with the convection that $\omega_{n}=0$ if the right hand side of the above identity is negative or equivalently

$$
\lambda_{n}^{(j)}=0
$$

if $n>1 / g$. Any way both the function $\alpha$ and the $\lambda_{n_{j}} j=1, \ldots, K$ depend essentially on the parameter $g$.

(iii) One easily verifies that $W_{N}: H_{N} \rightarrow H_{N}$ is a positive self-adjoint operator. Moreover, $\forall u \in H$ and $\forall F \in H_{N}$

$$
\langle F, F\rangle_{N}=0 \Rightarrow\left\langle u \otimes F, W_{N+1}(u \otimes F)\right\rangle_{N+1}=0
$$

This property ensures that $\forall u \in H$. The creation operator $a^{+}(u): H_{N} \rightarrow$ $H_{N+1}$ is well defined. For all $u \in H$, the annihilation operator is, by definition, $a(u): H_{N+1} \rightarrow H_{N}$ the adjoint of $a^{+}(u)$ for the scalar product $(\cdot)_{N}$. As usual we note $a_{i}^{+}=a^{+}\left(e_{i}\right), a_{i}=a\left(e_{i}\right) i=1 \ldots K$, where $\left\{e_{1} \ldots e_{K}\right\}$ is a fixed orthonormal basis of $H$. We shall denote $\Gamma_{I}\left(H,\left(W_{N}\right)_{N>1}, g\right)$ the standard interacting Fock space constructed in this way.

We will now prove that the Haldane exclusion $g$-statistics coincides with the vacuum statistics of the operators

$$
e^{-\beta H_{N}}=e^{-\beta N_{1}} \otimes \ldots \otimes e^{-\beta N_{K}}
$$

THEOREM: A system of identical particles obeying Haldane's exclusion statistics with statistical parameter $g$ is the standard IFS $\Gamma_{I}\left(H ;\left(W_{N}\right)_{N \geq i} ; g\right)$ over the Hilbert space of the single-particle state.

The corresponding creation and annihilation operators verify the following relations:

$$
\begin{gathered}
a_{i}\left|n_{1} \ldots n_{i} \ldots n_{K}\right\rangle=\frac{\lambda_{n_{i}}}{\lambda_{\left(n_{i}-1\right)}} \mid n_{1} \ldots \ldots n_{i}-1, \ldots, n_{K}> \\
{\left[a_{i}, a_{j}^{+}\right]=0 \quad \text { if } \quad i \neq j}
\end{gathered}
$$

We have also

$$
\begin{gathered}
{\left[a_{i}, a_{j}\right]=\left[a_{i}^{+}, a_{j}^{+}\right]=0 \quad \text { if } \quad i \neq j} \\
a_{i}^{+} a_{i}=\frac{\lambda_{N_{i}}}{\lambda_{\left(N_{i}-1\right)}}, \quad a_{i} a_{i}^{+}=\frac{\lambda_{\left(N_{i}+1\right)}}{\lambda_{N_{i}}} \\
a_{i}^{p+1}=a_{i}^{+(p+1)}=0 \quad, \quad \forall i, j=1 \ldots K \\
a_{i}|0\rangle=0 \quad, \quad \forall i=1 \ldots K
\end{gathered}
$$


Proof. To proof that Haldane's ES is realized in $\Gamma_{I}\left(H,\left(W_{N}\right)_{N \geq 1}, g\right)$ we just have to show that the partition function corresponding to this standard IFS is the same as the one of Haldane's ES. Generally for an ideal gas the partition function of $N$ particles is given by:

$$
Q_{N}=\operatorname{Tr}\left(e^{-\beta \mathcal{H}}\right)
$$

where $\mathcal{H}=\sum_{i=1}^{K} \varepsilon_{i} N_{i}$ is the Hamiltonian, $N_{i}$ is the number operator of the $i$-th species, $N=\sum_{i=1}^{K} N_{i}$ and the trace $T_{r}$ is expressed in any orthonormal basis of the Hilbert space of the states of the $N$ particles (see K. Huang: Statistical Mechanics, p. 196, p. 213). In our case this gives:

$$
\begin{aligned}
Q_{N} & =\sum_{n_{1}+\ldots n_{K}=N}\left\langle n_{1} \ldots n_{K}\left|e^{-\beta \mathcal{H}}\right| n_{1} \ldots n_{K}\right\rangle_{N} \\
& =\sum_{n_{1}+\ldots n_{K}=N} \alpha\left(n_{1} \ldots n_{K}\right) e^{-\beta \varepsilon n_{1}} \ldots e^{-\beta \varepsilon_{K} n_{K}}
\end{aligned}
$$

Given our choice of $\alpha\left(n_{1} \ldots n_{K}\right) Q_{N}$ has been shown in [2] to be exactly the partition function of Haldane's ES.

The relations (2)-(6) are easily verified.

\section{The IFS of Haldane's ES: the case of many species, many modes}

In the case $g=\frac{m}{p}, m>1$ and with $m$ and $p$ coprime, we can suppose that we have $K$ species of particles each of which with $m$ independent states, i.e. $H_{j}=\mathbb{C}^{m}$. We can also say that we have $p$ blocks each of which has $w$ species of particles with Hilbert space of dimension 1 for each species. Generalizing the above procedure we fix an ortho-normal basis $\left(e_{k}^{(j)}\right)$ of $H_{j}=\mathbb{C}^{m}$, we denote $a_{j, k}^{+}:=a^{+}\left(e_{k}^{(j)}\right)$ and we say that this creator generates the sub-species $(j, k)$ of the $j$-th species. We assume for simplicity that each species (and sub-species) has the same statistical parameter $g^{\prime}=1 / p$, but with essentially no changes in the construction below we could as well deal with the case in which the the $(j, k)$ sub-species has parameter $g_{j, k}^{\prime}=1 / p_{j, k}$. This would include the most general statistics considered by Haldane. With these notations we get:

1) For each species $j=1 \ldots K$ we have a standard IFS

$$
\Gamma_{j}=\Gamma_{j}\left(K,\left(W_{N_{j}}\right)_{N_{j} \geq 1}, \frac{1}{p}\right)=\oplus_{N_{j}} K_{N_{j}}
$$

of the type considered in section (5). More explicitly:

$$
K_{N_{j}}=\operatorname{span}\left\{a_{j, 1}^{+n_{j_{1}}} \ldots a_{j, m}^{+n_{j_{m}}}|0\rangle=\left|n_{j, 1}^{1}, \ldots, n_{j, m}^{m}\right\rangle: \sum_{i=1}^{m} n_{j i}=N_{j}\right\}
$$


2) The standard IFS for the whole population of $K$ species is:

$$
\begin{aligned}
\Gamma=\bigotimes_{j=1}^{K} \Gamma_{j} & =\bigotimes_{j=1}^{K}\left(\bigoplus_{N_{j}} K_{N_{j}}\right) \\
& =\bigoplus_{N \in \mathbb{N}}\left[\bigoplus_{N_{1}+\ldots+N_{K}=N} K_{N_{1}} \otimes K_{N_{2}} \otimes \ldots \otimes K_{N_{K}}\right] \\
& =\bigoplus_{N \in \mathbb{N}} \mathcal{H}_{N}
\end{aligned}
$$

where $\forall N$, the weight operator $W_{N}: H_{N} \rightarrow H_{N}$, defining the scalar product on $\mathcal{H}_{N}$ through the identity:

$$
(u, v)_{N}=\left\langle W_{N}(u), v\right\rangle
$$

$(\langle\cdot, \cdot\rangle$ being the usual scalar product $)$ is given by

$$
W_{N}=\sum_{N_{1}+\ldots+N_{K}=N}^{\oplus} W_{N_{1}} \otimes \ldots \otimes W_{N_{K}}
$$

and the operators $W_{N_{j}}$ are defined as in section (5).

REMARK. The above construction allows to realize any partition function of a system of identical particles, whose structure is given by the equation (1) by means of a standard IFS.

\section{References}

[1] Chaturvedi S. and Srinivasan V.: Microscopic Interpretation of Haldane's Semion Statistics, Phy. Rev. Lett. 78 (1997) p. 4316.

[2] Ilinski K.N., Gunn J.M.F., Ilinskaia A.V.: Fractional-dimensional Fock spaces, Second Quantization, and Dynamical Interaction of Particles with Haldane's Exclusion Statistics, Phy. Rev. B 53 (1996) p. 2615.

[3] Accardi L., Lu Y.G., Volovich I.: The QED Hilbert module and Interacting Fock spaces, Publications of IIAS (Kyoto) (1997).

[4] Accardi L., Bożejko M.: Interacting Fock spaces and Gaussianization of probability measures Infinite dimensional analysis, quantum probability and related topics, 2, N. 4 (1998) Volterra Preprint (1997) N. 321.

[5] Ha Z.N.C.: Exact Dynamical Correlation Functions of Calogero-Sutherland Model and One-Dimensional Fractional Statistics, Phy. Rev. Lett. 73 (1994) p. 1574 . 
[6] Haldane F.D.M.: "Fractional Statistics" in Arbitrary Dimensions: A Generalization of the Pauli Principle, Phy. Rev. Lett. 67 (1991) p. 937.

[7] Yong-Shi Wu: Statistical Distribution for Generalized Ideal Gas of Fractional Statistics Particles, Phy. Rev. Lett. 73 (1994) p. 922.

[8] Ilinski K.N., Gunn J.M.F.: Fractional Dimensional Fock space and Haldane's Exclusion Statistics: $\frac{q}{p}$ case and $t-J$ Model. Phy. Lett. A 210 (1996) p. 168.

[9] Ha Z.N.C.: Fractional statistics in one dimension: view from an exactly solvable model, Nucl. Phys. B 435 [FS] (1995) 604-636. 